\title{
Numerical and Data-Driven Modelling in Coastal, Hydrological and Hydraulic Engineering
}

\author{
Fangxin Fang (D)
}

Citation: Fang, F. Numerical and Data-Driven Modelling in Coastal, Hydrological and Hydraulic

Engineering. Water 2021, 13, 509. https://doi.org/10.3390/w13040509

Received: 5 February 2021

Accepted: 12 February 2021

Published: 16 February 2021

Publisher's Note: MDPI stays neutral with regard to jurisdictional claims in published maps and institutional affiliations.

Copyright: (C) 2021 by the author. Licensee MDPI, Basel, Switzerland. This article is an open access article distributed under the terms and conditions of the Creative Commons Attribution (CC BY) license (https:// creativecommons.org/licenses/by/ $4.0 /)$.
Applied Modelling and Computation Group, Department of Earth Science and Engineering, Imperial College London, Prince Consort Road, London SW7 2AZ, UK; f.fang@imperial.ac.uk

\section{Introduction}

This special issue aims at exploring advanced numerical techniques for real-time prediction and optimal management in coastal and hydraulic engineering. Numerical simulations of fluid dynamics have been indispensable in many applications relevant to physics and engineering.

Multiscale physical modelling: The grand challenge in modelling complex physical phenomena is to predict their characteristics and evolution with adequate accuracy and reliability. This has remained an open scientific problem for decades, unsurprisingly given the extreme disparity in the length- and time-scales involved, spanning several orders of magnitude. For example, climate modelling involves both basin scale and smaller scale features such as boundary currents, mixing, chemical interactions and transport, overflows, and mesoscale eddies. Over traditional structured mesh models, the use of adaptive unstructured meshes provides several profound and widely acknowledged advantages [1]. These include: the ability to conform accurately and efficiently to complex domain geometries (for example, bathymetry, a complex cityscape); the ability to dynamically adapt mesh resolution to improve the accuracy of model results, to capture detailed dynamics, or follow the physical processes.

Data assimilation - incorporating information from experiments and observations to reduce uncertainties in numerical prediction $[2,3]$. To achieve a predictive capability, numerical modelling often needs to be linked with experiments/monitoring. Data assimilation is a versatile methodology for estimating model uncertainties (parameters, model error, initial and boundary conditions, etc.). A variety of approaches have been used to facilitate data assimilation and include statistical interpolation methods, nudging data assimilation, Kalman filter, Ensemble Kalman filter, and variational (adjoint) methods. Optimal monitoring location is the key in data assimilation. Targeted observations represent a subcategory under adaptive observations where data collection is optimized to improve a particular forecast aspect (e.g., energy, health impact). The targeting approach incorporates dynamical information from numerical model predictions to identify when, where, and what types of observations would provide the greatest improvement to specific model forecasts at a future time [4]. Such targeted observations are important as they will allow the most effective use of available monitoring resources.

Data-driven modelling: Numerical models have benefited from the availability of high-resolution spatio-temporal data due to recent advances in measurement techniques [5], which make the studies of more complex flows possible. However, the computational cost involved in solving complex problems is intensive, which still precludes the development in these areas. Recently, data-driven modelling has gained popularity in rapidly predicting nonlinear fluid flows. Various studies have shown that machine learning methods have potential for capturing non-linear subgrid processes. Reichstein et al. [6] suggest that future models should integrate physical-based modelling and machine learning approaches. Such combination will be the optimal way by which physical modelling results can provide 
dynamic understanding from the governing equations, while data-driven modelling results may find some patterns that are not expected from physical modelling.

The Special Issue of Water journal comprises five original papers covering the aspects of the above-mentioned challenges. Overall, the authors focused on different topics, such as, multiscale modelling [7], data assimilation [8], adaptive observation [9], machine learning [10], and application [11].

\section{Author Contributions}

Juznetsov et al. [7] presented the results with a new 3D unstructured mesh finite volume coastal model, FESOM-C [12]. Over existing structured mesh models, FESOM-C with hybrid meshes (quadrilaterals and triangles) is geometrically flexible and allows the resolution of the mesh to be varied (within reasonable limits) in both the horizontal and vertical, letting the mesh contour complex coastlines. Variable horizontal resolution enables the use of coarser meshes in open sea regions but more refined ones in shallow areas to resolve important small-scale processes such as wetting and drying, sub-mesoscale eddies, or sub-mesoscale dynamics of steep coastal fronts. In this work, the FESOM-C model was applied to an area of the south-eastern North Sea and its performance was evaluated by comparing the results against observations. With use of mixed unstructured meshes, the salinity and temperature gradients, as well as frontal dynamics, can be captured well.

Du et al. [8] explored the capability of ensemble Kalman filter (EnKF) in its applications. EnKF has its potential for efficient use on parallel computers with large-scale geophysical models. EnKF techniques are widely used in operational modelling. The EnKF is based on a Monte Carlo approach, using an ensemble of model representations to build up the necessary statistics [13]. A background error covariance is computed using an ensemble of forecasts, with the current analysis ensemble serving as initial conditions. However, the introduction of perturbations into background variables may break the physical conservation law. One of challenges in EnKF is how to maintain the physical features of variables after inducing the initial perturbations. To address this issue, Du et al. [8] proposed a multivariate balanced initial ensemble generation method based on the multivariate empirical orthogonal function (MEOF) method. The Local Ensemble Transform Kalman Filter in combination with the MEOF based balanced perturbation scheme was applied for improving accuracy of atmospheric general circulation modelling. The study case was the six-hour model forecast from 1 January to 31 March 2004. The prognostic model variables were temperature, surface pressure, wind velocity, and specific humidity. The results obtained from their work suggested that the ensembles integrated from the initial ensemble generated from the MEOF-based perturbations maintain a much more reasonable spread and more reliable horizontal correlation than those from the randomly perturbed initial fields.

Fattorni and Brandini [9] presented their recent work on adaptive observation strategy. Due to expensive field-deployed resources, there is a need to optimally place observations that will maximally improve the accuracy of numerical solutions at forecast times. The optimal observation network could be adapted for a wide range of forecasting goals, and it could be adapted either by allocating existing observations differently or by adding observations from programmable platforms to the existing network. Fattorni and Brandini [9] proposed the observation strategies based on singular value decomposition (SVD). In their work, SVD was used for identifying the areas where maximum error growth occurred, and a correlation analysis was used to limit redundant observations. The case study was Double Gyre, a well-known idealized case to reproduce the seasonal and interannual oscillations of the large-scale circulation in the ocean, useful for the climate system predictability. The results indicated that optimal observation strategies can provide effective and efficient data assimilation for improving predictive accuracy.

He et al. [10] explored the potential of deep learning techniques in emulating the process-based Martinez Boundary Salinity Generator in simulating downstream salinity boundary for the Sacramento-San Joaquin Delta of California, United States. In their work, 
the multilayer perceptron, long short-term memory network, and convolutional neural network-based models were used. The training datasets were from 1991 to 2002 while validation datasets were from 2003 to 2014 . The results obtained in this work showed that deep learning neural networks can provide competitive or superior results compared to the process-based model, especially during extreme (i.e., wet, dry, and critical) years.

Lastly, Gao et al. [11] presented their study of transport time scales (TTS) for a HyperTidal Estuary. The water exchange processes and transport time scales are important factors in governing tracer transport, water quality, and the ecosystem of the basin [14]. The study site chosen in this work was the Severn Estuary, one of the largest estuarine basins in the UK, with typical spring and neap tidal ranges of $13.5 \mathrm{~m}$ and $6.5 \mathrm{~m}$, respectively. The aim of this study was to investigate the residence and exposure times of the Severn Estuary in parallel, and to characterize the transport time scales for a hyper-tidal estuary. The results obtained from their work suggested that for all flow and tide conditions, the exposure times are significantly greater than the residence times. That is, there is a high possibility for water and/or pollutants to re-enter the Severn Estuary after leaving it on an ebb tide. The fractions of water and/or pollutants re-entering the estuary for spring and neap tide conditions are very high, with $0.75-0.81$ for neap tides, and $0.79-0.88$ for spring tides.

In conclusion, this special issue presents recent studies on numerical and data-driven modelling and applications to atmosphere, ocean, coastal, and hydrological engineering.

Funding: This research received no external funding.

Institutional Review Board Statement: Not applicable.

Informed Consent Statement: Not applicable.

Data Availability Statement: Data sharing not applicable.

Conflicts of Interest: The authors declare no conflict of interest.

\section{References}

1. Piggott, M.D.; Farrell, P.E.; Wilson, C.R.; Gorman, G.J.; Pain, C.C. Anisotropic mesh adaptivity for multi-scale ocean modelling. Philos. Trans. R. Soc. A Math. Phys. Eng. Sci. 2009, 367, 4591-4611. [CrossRef] [PubMed]

2. Ghil, M.; Malanotte-Rizzoli, P. Data assimilation in meteorology and oceanography. Adv. Geophys. 1991, 33, 141-266.

3. Kalnay, E. Atmospheric Modeling, Data Assimilation and Predictability; Cambridge University Press: Cambridge, UK, 2003.

4. Daescu, D.N.; Carmichael, G.R. An adjoint sensitivity method for the adaptive location of the observations in air quality modeling. J. Atmos. Sci. 2003, 60, 434-450. [CrossRef]

5. Taira, K.; Hemati, M.S.; Brunton, S.L.; Sun, Y.; Duraisamy, K.; Bagheri, S.; Dawson, S.T.; Yeh, C.A. Modal analysis of fluid flows: Applications and outlook. AIAA J. 2020, 58, 998-1022. [CrossRef]

6. Reichstein, M.; Camps-Valls, G.; Stevens, B.; Jung, M.; Denzler, J.; Carvalhais, N. Deep learning and process understanding for data-driven Earth system science. Nature 2019, 566, 195-204. [CrossRef] [PubMed]

7. Kuznetsov, I.; Androsov, A.; Fofonova, V.; Danilov, S.; Rakowsky, N.; Harig, S.; Wiltshire, K.H. Evaluation and Application of Newly Designed Finite Volume Coastal Model FESOM-C, Effect of Variable Resolution in the Southeastern North Sea. Water 2020, 12, 1412. [CrossRef]

8. Du, J.; Zheng, F.; Zhang, H.; Zhu, J. A Multivariate balanced initial ensemble generation approach for an atmospheric general circulation model. Water 2021, 13, 122. [CrossRef]

9. Fattorini, M.; Brandini, C. Observation strategies based on singular value decomposition for ocean analysis and forecast. Water 2020, 12, 3445. [CrossRef]

10. He, M.; Zhong, L.; Sandhu, P.; Zhou, Y. Emulation of a process-based salinity generator for the sacramento-san joaquin delta of california via deep learning. Water 2020, 12, 2088. [CrossRef]

11. Gao, G.; Xia, J.; Falconer, R.A.; Wang, Y. Modelling study of transport time scales for a hyper-tidal estuary. Water 2020, $12,2434$. [CrossRef]

12. Fofonova, V.; Androsov, A.; Sander, L.; Kuznetsov, I.; Amorim, F.; Hass, H.C.; Wiltshire, K.H. Non-linear aspects of the tidal dynamics in the Sylt-Rømø Bight, south-eastern North Sea. Ocean Sci. 2019, 15, 1761-1782. [CrossRef]

13. Evensen, G. The ensemble Kalman filter: Theoretical formulation and practical implementation. Ocean Dyn. 2003, 53, 343-367. [CrossRef]

14. Uncles, R.J.; Stephensa, J.A.; Smithb, R.E. The dependence of estuarine turbidity on tidal intrusion length, tidal range and residence time. Cont. Shelf Res. 2002, 22, 1835-1856. [CrossRef] 